\title{
Adopting Agile Practices When Developing Software for Use in the Medical Domain
}

\author{
Martin McHugh \\ Technological University Dublin, martin.mchugh@tudublin.ie \\ Fergal McCaffery \\ Dundalk Institute of Technology, fergal.mccaffery@dkit.ie \\ Valentine Casey \\ Dundalk Institute of Technology, val.casey@dkit.ie
}

Follow this and additional works at: https://arrow.tudublin.ie/scschcomart

Part of the Computer Sciences Commons

\section{Recommended Citation}

Mc Hugh, M., Mc Caffery, F. \& Casey, V. (2014) Adopting Agile Practices when developing software for use in the Medical Domain, Journal of Software Evolution and Process, Wiley, Vol. 26, Issue 5, pp. 504-512.

doi:10.1002/smr.1608

This Article is brought to you for free and open access by the School of Computer Sciences at ARROW@TU Dublin. It has been accepted for inclusion in Articles by an authorized administrator of ARROW@TU Dublin. For more information, please contact arrow.admin@tudublin.ie, aisling.coyne@tudublin.ie,gerard.connolly@tudublin.ie.

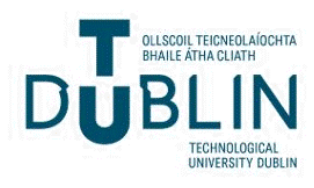


Dublin Institute of Technology

ARROW@DIT

2013-05-01

\title{
Adopting Agile Practices when developing software for use in the Medical Domain
}

\author{
Martin Mc Hugh \\ Fergal McCaffery \\ Valentine Casey
}

Follow this and additional works at: http://arrow.dit.ie/scschcombk

Part of the Computational Engineering Commons

This Article is brought to you for free and open access by the School of

Computing at ARROW@DIT. It has been accepted for inclusion in Books/

Book Chapters by an authorized administrator of ARROW@DIT. For

more information, please contact yvonne.desmond@dit.ie,

arrow.admin@dit.ie. 


\title{
Adopting Agile Practices when developing software for use in the Medical Domain
}

\author{
Martin McHugh $^{* \dagger}$, Fergal McCaffery and Valentine Casey \\ Regulated Software Research Centre. Dundalk Institute of Technology, Dundalk, Co. Louth, Ireland
}

\section{SUMMARY}

Non-safety critical software developers have been reaping the benefits of adopting agile practices for a number of years. However, developers of safety critical software often have concerns about adopting agile practices. Through performing a literature review, this research has identified the perceived barriers to following agile practices when developing medical device software. A questionnaire based survey was also conducted with medical device software developers in Ireland to determine the barriers to adopting agile practices. The survey revealed that half of the respondents develop software in accordance with a plan driven software development lifecycle and that they believe that there are a number of perceived barriers to adopting agile practices when developing regulatory compliant software such as: being contradictory to regulatory requirements; insufficient coverage of risk management activities and the lack of up-front planning. In addition, a comparison is performed between the perceived and actual barriers. Based upon the findings of the literature review and survey, it emerged that no external barriers exist to adopting agile practices when developing medical device software and the barriers that do exists are internal barriers such as getting stakeholder buy in.

KEY WORDS: Agile Methods, Medical Device, V-Model, Plan Driven, XP, Medical Device Software

\section{INTRODUCTION}

The popularity of agile practices is on the rise [1]. Agile practices appear to offer a "silver bullet" [2] for all of the problems associated with traditional plan driven software development lifecycles. A number of surveys have been completed which reinforce this believe [3, 4]. However, a large amount of research that has been conducted into the success of adopting agile practices is broad and does not expressly focus on specific domains within the software development industry i.e. safety critical software development.

Non-safety critical software is developed in accordance with a customer's requirements, but safety critical software must be developed in accordance with both customer requirements and national and/or international regulatory constraints. These regulatory constraints are dictated by the region in which it is planned to market the software, be it standalone or embedded within a hardware device. For example, if a medical device is to be marketed in the United States (US) it must be developed in accordance with the Food and Drug Administration (FDA) quality regulations, guidance documents and approved standards [5, 6]. Software developed for use within safety critical domains is typically developed in accordance with the Waterfall Model or V-Model software development lifecycles [6, 7]. These lifecycles are defined by upfront design with high importance placed upon the

\footnotetext{
Correspondence to: Martin Mc Hugh, Regulated Software Research Centre, Department of Computing and Mathematics, Dundalk Institute of Technology, Dundalk Co. Louth Ireland

${ }^{\dagger}$ Email: martin.mchugh@dkit.ie
} 
production of documentation [6]. These models produce the necessary deliverables required to achieve regulatory conformance.

Our research is focused on the development of software for use within the medical device domain. Regulatory requirements and development standards such as [8,9] do not dictate the use of a particular lifecycle when developing medical device software. In fact they state that medical device software can be developed using a traditional, iterative and/or evolutionary approach. Despite this, medical device software developers typically develop software in accordance with the V-Model [7]. Whilst the V-Model produces necessary deliverables such as traceability between requirements and all stages of the software development lifecycle [10] it is seen as being rigid and inflexible in the event of a change once development has begun [11].

This research was initiated by performing a literature review. One of the objectives of undertaking this literature review was the identification of the perceived barriers to adopting agile practices when developing medical device software. Also as part of this research a questionnaire based survey was conducted amongst medical device software developers in Ireland. The aim of this survey was to evaluate the findings of the literature review and to learn what the actual barriers are to adopting agile practices when developing medical device software. The findings of the literature review revealed that there is a slow rate of adoption of agile practices when developing medical device software and that there are a number of perceived barriers to adopting agile practices when developing medical device software [12].

The remainder of this paper is structured as follows: Section 2 provides information as to our on-going research in this area and how this aspect of our work fits into this research. In section 3, we outline related work which was identified through the literature review. Section 4 details the perceived barriers to selecting and implementing agile practices when developing medical device software based on the results from our literature review. Section 5 outlines the approach taken by questionnaire based survey conducted amongst medical device manufacturers in Ireland. Section 6 provides the results of the survey. Within section 7 a comparison is performed between the perceived barriers and the actual barriers to adopting Agile practices and finally within section 8 conclusions of this research are presented.

\section{RESEARCH OBJECTIVES}

As part of this on-going research the following research questions have been identified:

1. What are the issues associated with developing medical device software?

2. What are the issues with developing medical device software using a traditional software development lifecycle?

3. Can agile practices be used to develop medical device software?

4. If agile practices can be used to develop medical device software, how must they be incorporated within the existing lifecycle in order to meet regulatory requirements?

The results from the research outlined in this paper will be used to help address the third research question. By identifying actual barriers to the adoption of agile practices specific practices can be discounted and the remaining agile practices can be evaluated for suitability. These research questions were formed following the completion of the literature review. This literature review began by broadly looking at generic software development lifecycles. It 
became clear through this portion of the literature review that the generic software development industry is greatly benefitting from the adoption of agile software development methods. The focus of the literature review moved to the development of safety critical software and then onto the development of software in the medical device industry. Following this phase of the literature review, research was conducted into agile software development. This involved examining mainstream methodologies such as Scrum and XP. Once this was completed we then focused upon the adoption of agile practices in the development of safety critical software. Finally, we considered the adoption of agile practices in the development of medical device software. This literature revealed a number of perceived barriers to adopting agile practices when developing medical device software.

Following the literature review a questionnaire based survey was conducted amongst medical device software developers in Ireland. The objective of this survey was to evaluate the findings of the literature review and to learn what the actual barriers to adopting agile practices are.

\section{RELATED RESEARCH}

In October 2012 the Association for the AAMI released a Technical Information Report (TIR) known as AAMI TIR 45:2012 [13]. The committee which developed the TIR consisted of industry experts and FDA staff. The AAMI recognised the shift in the generic software development industry towards more agile practices. However, they identified that the available information with regard to implementing agile methods when developing medical device software was hard to understand and the objective of the TIR is to provide clear guidance on which practices of agile software development are suited to the development of medical device software. The TIR also provides recommendations for complying with international standards and FDA guidance documents when using agile practices to develop medical device software.

When considering agile development methods, development organisations will typically look at the agile manifesto. The agile manifesto [14] contains 4 key statements and 12 practices. The 4 key statements appear to be contradictory to the values of regulated software development. These 4 key statements are:

\section{"Individuals and Interactions over process and tools \\ Working Software over comprehensive documentation \\ Customer Collaboration over contract negotiation \\ Responding to change over following a plan"}

However these values need not be interpreted as being contradictory to the development of medical device software. The AAMI TIR states;

"they (the 4 key values) can be aligned to be complimentary, resulting in a quality management systems that produces high-quality medical device software"

As discussed, medical device software developers in both the EU and the US are strongly recommended to follow IEC 62304 in the development of regulatory compliant software. As this is the case, the AAMI mapped each of the identified suitable agile practices to the appropriate stage of development in IEC 62304. The TIR focuses on a number of areas in 
which agile software development practices are suited when developing medical device software. These areas include:

- Planning;

- Team Structure and Collaboration;

- Product Definition and Requirements documentation;

- Software Architecture;

- Detailed Design;

- Implementation and Unit Verification;

- Integration and Integration Testing;

- Software System Testing;

- Software Release;

- Configuration Management and Change Management;

- Corrective and Preventative Action.

The TIR successfully maps practices performed as part of agile software development techniques to each of these stages of development. To accompany this TIR, a number of case studies have emerged from medical device software organisations which have successfully used agile practices within their organisation. These organisations include, Abbott Diagnostics [15], Medtronic [16] and Cochlear [17]. A common trend appeared in each of the case studies. Each organisation recognised that no single agile methodology such as Scrum or XP could be wholly followed in the development of medical device software. Instead, these organisations look at the practices contained with the individual methodologies and selected practices most suited to their organisational need. Additionally whilst these organisations selected appropriate practices to follow, for varying reasons, they choose to integrate the selected practices with the traditional plan driven SDLC currently in place.

\section{PERCEIVED BARRIERS TO AGILE ADOPTION}

Software developed for use in or as a medical device must meet the regulatory requirements of the region where the device is being marketed. As a result many of the barriers to adopting agile practices, in developing medical device software are associated with regulatory controls [18]. The focus of this research is the identification of the perceived barriers that have a direct impact on the development process of medical device software and the implementation of agile methods in this context. Additional barriers do exist, but a number of these are organisational barriers that do not have a direct impact on the development of medical device software. An example of such an organisational barrier is that Human Resource policies and processes do not cater for the requirements of an Agile team [19]. The literature review identified the following perceived barriers to the adoption of agile practices when developing medical device software.

The FDA General Principles of Software Validation (GPSV) [9] require manufacturers to explicitly document requirements prior to implementation and test procedures [20]. This would appear to be an apparent barrier to adopting agile practices as one of the fundamental principles of the agile Manifesto [21] is "working software over comprehensive documentation". Combined with this, another central principle of agile software development is that requirements are fluid and changes in requirements can be easily accommodated and are even welcomed throughout a development project [22]. Without fully refining 
requirements prior to the beginning of a project the process of traceability can be difficult and traceability between requirements and all stages of development is required by the FDA [23].

As safety critical software, such as medical device software, can place patients, clinicians and third parties at potential risk, medical device software developers must perform adequate risk management activities to ensure the software they are developing is safe and reliable. Boehm and Turner [19] suggest that risk management activities can be a barrier to adoption as agile practices do not provide sufficient guidance as how to perform the necessary risk management activities.

Another perceived barrier to adopting agile practices is that software developed using agile practices is of a lower quality than software developed following traditional plan driven lifecycles [19]. As medical device software is safety critical it must be developed to the highest quality possible.

Agile methodologies such as XP recommend short releases with continuous feedback [24]. When developing medical device software, it is not possible to release incomplete software and await feedback as the software must be fully tested and working before it is used in patient treatment [25].

An additional potential barrier to adopting agile practices in the development of both safety critical and non-safety critical software is the loss of management control. Agile methodologies recommend that development teams are self-organising. This process of selforganising teams removes some of the decision making powers from management [26]. This may result in a loss of management control and for agile practices to succeed organisational support is required [27].

\section{SURVEY OF MEDICAL DEVICE SOFTWARE DEVELOPERS}

In order to gain an understanding of the barriers to the adoption of agile practices we performed a questionnaire based survey with medical device software development organisations within Ireland. The literature review outlined in section 1 identified the perceived barriers to agile adoption and the survey results have been used to determine actual barriers to adopting agile practices when developing medical device software.

Within Ireland there are approximately 160 medical device manufacturers [28]. No research has been conducted to date to suggest how many of these organisations develop medical device software. As a result when determining sufficient sample size, a decision was made to assume all of these organisation develop medical device software. Using sample size equations, a sufficient sample size was determined to be twenty organisations.

As a result of this, the survey was conducted amongst twenty medical device software development organisations in Ireland with multiple responses from each organisation. These organisations ranged from small indigenous manufacturers to large multinational manufacturers. The devices produced by theses manufacturers range from Class I - Low Risk to Class III - High Risk ${ }^{\ddagger}$ products. The primary goal of this survey was to gain a deeper insight into the medical device software development industry to further assist with our on-

\footnotetext{
‡ This safety classification is defined by the European Council Medical Device Directive 93/42/EEC. Class I devices are deemed to pose low risk to patients, users and third parties and Class III devices are deemed to pose potentially life threatening risk to patients, users and third parties.
} 
going research. Participants who took part in the survey included all levels of the development team and internal stakeholders such as managers and senior management.

\subsection{RESEARCH METHODOLOGY}

When developing the survey a number of areas needed to be considered. Once the objectives had been clearly defined, a mechanism to elicit the required information was needed. The survey was constructed in accordance with Sapsford [29]. Sapsford provides a number of questions which should be asked prior to constructing a survey:

- What's the problem?

- What kind of answer am I looking for?

- What kind of an argument might lead from the question to the answer?

- What kind of evidence will I need to sustain this kind of argument? [29, p.13].

Based upon these questions the survey was constructed. Based upon this method suggested by Sapsford and by Frink [30] a bottom up approach for question formulation was used. Both Sapsford and Frink advocate looking at which information you want to receive from the survey and developing the survey questions accordingly. The process of developing the survey questions was an iterative one. The iterative lifecycle of the question formulation is shown in the figure 1 .

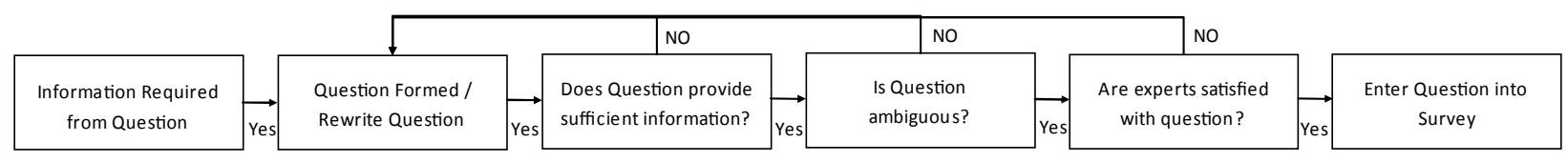

Figure 1 Iterative Process of Question Formulation

Figure 1 shows the transition from the data required from the question through to being entered into the survey. This process was completed for each question in the survey. The benefit to this method of question construction is that each question has been validated before being entered into the survey. Once the survey was completed it was distributed via web link.

\section{RESULTS OF THE SURVEY}

The first piece of key information obtained by the survey was which software development lifecycle the organisations are following. As part of this on-going research, recommendations will be made as to how adopting agile practices can resolve problems associated with the current lifecycle being followed.

Following on from this question, participants were provided with a list of activities that are required to be completed in the development of safety critical software. Participants were requested to rate how much importance they place on each of these activities and to rate how effective they deem their organisation to be at performing these activities. The objective of this question was to understand which areas of safety critical software development are being performed most effectively. Again as part of this on-going research, information is being collected that will identify which stages of development pose the most difficulty to medical device software developers. This information will eventually be used to help answer research questions 1 and 2 . 
Finally participants were asked a series of questions relating to agile software development. Participants were provided with a list of 10 potential barriers to agile adoption when developing medical device software. The goal of this question was to evaluate the findings of the literature review. Participants where then asked what the actual barriers were within their own organisation to the adoption of agile methods. This question was used to establish what these barriers are and if commonality could be identified across organisations as to what actual barriers exist in relation to agile adoption. A barrier being defined as an actual barrier does not imply that the barrier is insurmountable. To maximise the amount of relevant information gathered, space was provided for the respondents to add additional information and/or comments for each question as they deemed necessary.

The survey identified that $50 \%$ of the organisations are developing software in accordance with the V-Model. An important finding was that another $25 \%$ of the organisations are developing medical device software in accordance with agile practices. The remaining $25 \%$ of organizations are developing software in accordance with other development lifecycles such as the Waterfall model. As part of the survey, respondents were asked what they believe to be the barriers to agile adoption. The survey revealed that $25 \%$ of respondents reported "Lack of Documentation" as a barrier to agile adoption. In addition 25\% of respondents reported "Regulatory Compliance", whilst $16 \%$ of respondents reported "Lack of Up-Front planning" and $17 \%$ of respondents reported "Insufficient coverage of risk management activities" as barriers to agile adoption. These results were consistent with the findings from the literature review.

Finally, respondents of the survey were asked what the actual barriers to adopting agile practices are within their organisation. Of the respondents 50\% reported "Lack of Experience", $33 \%$ reported that "having to change the existing lifecycle as a barrier to agile adoption", 16\% reported "Management Opposed to Change "and 16\% reported team size as a barrier to agile adoption. A further $17 \%$ reported that getting stakeholder buy in as a barrier and $17 \%$ reported the level of retraining required as another barrier to agile adoption.

\section{COMPARISON BETWEEN PERCEIVED AND ACTUAL BARRIERS}

Table 1 presents a list of the perceived barriers based on our literature review and the actual barriers to agile adoption when developing medical device software based on our survey results. It can be seen in table 1 that a number of the perceived barriers are also actual barriers to using agile practices when developing medical device software. An important point to emerge from our research is how requirements can be identified and successfully managed in the context of utilising agile practises. Regulatory bodies require medical device software developers to document requirements prior to development. These requirements are then used during the development stages to provide traceability. Agile principles dictate that requirements must be fluid throughout a development project and this can be seen as a barrier as we have outlined in section 4.

However, regulatory bodies do recognise the acceptability of what can be termed an agile approach to requirements. The FDA GPSV states [9]: 
"Most software development models will be iterative. This is likely to result in several versions of both the software requirement specification and the software design specification. All approved versions should be archived and controlled in accordance with established configuration management procedures".

Table 1 Perceived and Actual Barriers to Agile adoption

\begin{tabular}{|c|c|c|}
\hline Perceived Barriers & & Actual Barriers \\
\hline $\begin{array}{ll}\text { - } & \text { Regulatory Control } \\
\text { - } & \text { In-sufficient coverage } \\
& \text { Management Activities } \\
\text { - } & \text { Requirements Management } \\
\text { - } & \text { Traceability issues } \\
\text { - } & \text { Loss of management control } \\
\text { - } & \text { Lower quality software }\end{array}$ & Risk & $\begin{array}{ll}\text { - } & \text { Regulatory Control } \\
\text { - } & \text { In-sufficient coverage of Risk } \\
& \text { Management Activities } \\
\text { - } & \text { Lack of up-front planning } \\
\text { - } & \text { Lack of documentation } \\
\text { - } & \text { Management opposed to change } \\
\text { - } & \text { Team size } \\
\text { - } & \text { Modification of existing lifecycle } \\
& \\
\text { - } & \text { Lack of Experience using agile } \\
\text { - } & \text { Getting Stakeholder Buy In } \\
\text { - } & \text { Level of Retraining Required }\end{array}$ \\
\hline
\end{tabular}

This emerged from our detailed analysis of the relevant regulations, standards and guidance documents. This was not evident from the published academic literature in this area, in fact the opposite was the case as we have stated.

This provides an example of how the perceived barriers to agile adoption can be overcome. Further research will be undertaken to evaluate and determine how each of the other barriers identified can be addressed by employing specific agile practices or by integrating agile practices with a plan driven lifecycle.

\section{CONCLUSIONS}

Medical device software must be developed in accordance with the appropriate regulatory requirements and development standards of the region into which it is being marketed. For the US, these regulations are dictated by the FDA. They recommend medical device development organisations follow IEC 62304 when developing regulatory complaint software. Neither the regulations or standards dictate which SDLC should be followed then developing regulatory compliant software; however medical device software is typically developed in accordance with the V-Model. The V-Model suffers the same problems as with all sequential plan driven SDLC such as a large emphasis being placed on up-front requirements and the difficulty accommodating a change once development has begun. To overcome these challenges, this research is exploring the possibility of adopting agile practices when developing medical device software in conjunction with a plan driven software development lifecycle.

To understand whether it was feasible to adopt agile practices a literature review of development standards and regulations was performed. This literature review revealed there to be no direct barriers by regulatory bodies or development standards to adopting agile methods when developing medical device software. To validate the findings of this literature review, a questionnaire based survey was performed with medical device development organisations. This survey revealed two types of barriers, perceived and actual. Perceived barriers are barriers which organisations feel exist, but through the literature review 
performed are in fact only superficial. Actual barriers are barriers which exist which directly prevent the adoption of agile methods when developing medical device software. Examples of perceived barriers include: Regulatory Control; Traceability Issues and Lower Quality Software. Examples of the actual barriers include: Lack of Stakeholder Buy-In, Lack of Experience using agile and the level of retraining required. The literature review, questionnaire based survey and AAMI TIR 45:2012 act as evidence that there are no external barriers to adopting agile methods when developing medical device software and that barriers that do exist, are primarily in-house barriers within the organisation which can be overcome.

\section{ACKNOWLEDGEMENTS}

This research is supported by the Science Foundation Ireland (SFI) Stokes Lectureship Programme, grant number 07/SK/I1299, the SFI Principal Investigator Programme, grant number 08/IN.1/I2030 (the funding of this project was awarded by Science Foundation Ireland under a co-funding initiative by the Irish Government and European Regional Development Fund), and supported in part by Lero - the Irish Software Engineering Research Centre (http://www.lero.ie) grant 10/CE/I1855.

\section{REFERENCES}

[1] K. Conboy and B. Fitzgerald, "Method and developer characteristics for effective agile method tailoring: A study of XP expert opinion," ACM Trans. Softw. Eng. Methodol, vol. 20, pp. 1-30, 2010.

[2] F. P. Brooks, "No Silver Bullet Essence and Accidents of Software Engineering," Computer, vol. 20, pp. 10-19, 1987.

[3] VersionOne, "State of Agile Survey - The Stage of Agile Development," 2010.

[4] S. Ambler. (2008). Agile Adoption Rate Survey Results: February 2008 Available: http://www.ambysoft.com/surveys/agileFebruary2008.html\#Downloads [Last accessed: 23/09/2012]

[5] F. McCaffery, J. Burton, V. Casey, and A. Dorling, "Medical Device Industry: Software Process Improvement," in Encyclopedia of Software Engineering, P. Laplanye, Ed., ed London: CRC Press Francis Taylor Group, 2010, pp. 528-540.

[6] X. Ge, R. F. Paige, and J. A. McDermid, "An Iterative Approach for Development of Safety-Critical Software and Safety Arguments," presented at the Agile 2010, Orlando, Florida, 2010.

[7] K. Bulska and J. Gorski, "Applying Agile Practices to the development of Safety-Critical Software," presented at the ICT Young 2011, Scientific Booklets of Faculty of Electronics, Telecommunications and Informatics, Gdańsk University of Technology, 2011.

[8] AAMI, "ANSI/AAMI/IEC 62304, Medical device Software - Software life cycle processes," ed. Association for the Advancement of Medical Instrumentation, 2006.

[9] FDA, "General Principles of Software Validation: Final Guidance for Industry and FDA Staff," ed: Centre for Devices and Radiological Health, 2002.

[10] F. McCaffery, V. Casey, M. Sivakumar, G. Coleman, P. Donnelly, and J. Burton, "Medical Device Software Traceability," in Software and Systems Traceability, J. Cleland-Huang, O. Gotel, and A. Zisman, Eds., ed: Springer-Verlag, 2012.

[11] N. M. A. Munassar and A. Govardhan, "A Comparison Between Five Models Of Software Engineering," IJCSI International Journal of Computer Science Issues, vol. 7, pp. 94-101, 2010.

[12] M. McHugh, O. Cawley, F. McCaffery, I. Richardson, and X. Wang, "An Agile V-Model for Medical Device Software Development to Overcome the Challenges with Plan-Driven Software Development Lifecycles," presented at the Software Engineering in Healthcare Workshop at the 35th International Confernence on Software Engineering (ICSE), San Francisco CA, 2013.

[13] AAMI, "AAMI TIR45:2012 -- Guidance on the use of agile practices in the development of medical device software," 2012.

[14] R. C. Martin, Agile Software Development - Principles, Patterns and Practices: Prentice Hall, 2003.

[15] R. Rasmussen, T. Hughes, J. R. Jenks, and J. Skach, "Adopting Agile in an FDA Regulated Environment," presented at the Agile Conference, 2009. AGILE '09. , Chicago, IL 2009. 
[16] K. Weyrauch, "What Are We Arguing About? A Framework for Defining Agile in our Organization," presented at the Proceedings of the conference on AGILE 2006, 2006.

[17] P. A. Rottier and V. Rodrigues, "Agile Development in a Medical Device Company," presented at the Proceedings of the 11th AGILE Conference. AGILE '08., Girona, Spain, 2008.

[18] S. Mishra and H. R. Weistroffer, "Issues with Incorporating Regulatory Compliance into Agile Development: A Critical Analysis," presented at the Southern Association for Information Systems (SAIS), 2008.

[19] B. Boehm and R. Turner, "Management Challenges to Implementing Agile Processes in Traditional Development Organizations," IEEE Software, vol. 22, pp. 30-39, 2005.

[20] D. Vogel, "Agile Methods: Most are not ready for prime time in medical device software design and development," DesignFax Online, 2006.

[21] M. Fowler and J. Highsmith. (2001) The Agile Manifesto. Software Development: The Lifecycle starts here.

[22] B. Boehm and R. Turner, Balancing Agility and Discipline: A Guide for the Perplexed: AddisonWesley, 2003.

[23] L. Weiguo and F. Xiaomin, "Software Development Practice for FDA-Compliant Medical Devices," presented at the International Joint Conference on Computational Sciences and Optimization, 2009. , Sanya, Hainan, 2009.

[24] P. Abrahamsson, O. Salo, J. Ronkainen, and J. Warsta, "Agile Software Development Methods Review and Analysis," presented at the ESPOO 2002, 2002.

[25] FDA, "Title 21--Food and Drugs Chapter I --Food and Drug Administration Department of Health and Human Services subchapter h--Medical Devices part 820 Quality System Regulation," ed: U.S. Department of Health and Human Services, 2007.

[26] N. B. Moe, T. Dingsoyr, and T. Dyba, "Understanding Self-Organizing Teams in Agile Software Development," presented at the Conference on Software Engineering, 2008. ASWEC 2008. 19th Australian Perth, Australia, 2008.

[27] O. Salo and P. Abrahamsson, "Integrating agile software development and software process improvement: a longitudinal case study," presented at the International Symposium on Empirical Software Engineering 2005.

[28] M. McHugh, "Medical Device Sector in Ireland," Network Enterprise Europe, 2010.

[29] R. Sapsford, Survey Research 2nd ed. London: Sage Publications, 2007.

[30] A. Frink, The Survey Handbook: Sage Publications, Inc;, 2003.

\section{AUTHORS’ BIOGRAPHY}

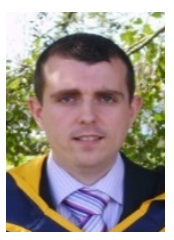

Martin Mc Hugh received his B.Sc. (Hons.) in Information Technology Management in 2005 and M.Sc. in Computer Science in 2009, from Dundalk Institute of Technology. He is now undertaking research for his Ph.D. in the area of software process improvement for medical devices with emphasis on the usage of agile practices when developing medical device software, as part of the Regulated Software Research Centre in Dundalk Institute of Technology.

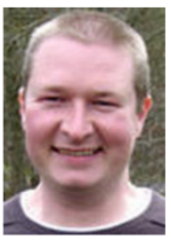

Dr Fergal Mc Caffery is the leader of the Regulated Software Research Centre in Dundalk Institute of Technology and a member of Lero. He has been awarded Science Foundation Ireland funding through the Stokes Lectureship, Principal Investigator and CSET funding Programmes to research the area of software process improvement for the medical device domain. Additionally, he has received EU FP7 and Enterprise Ireland Commercialisation research funding to improve the effectiveness of embedded software development environments for the medical device industry.

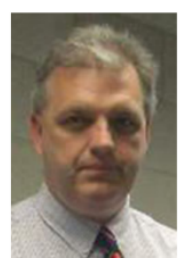

Dr Valentine Casey is a Senior Researcher with the Regulated Software Research Centre in Dundalk Institute of Technology. His previous roles include Senior Lecturer and Research Area Leader at Bournemouth University, Researcher with Lero - the Irish Software Engineering Research Centre at the University of Limerick where he also lectured. He has over 20 years' experience in the software industry. He has also provided consultancy services focusing on software process improvement and software testing in the financial and telecom sectors. 